\title{
AVALIAÇÃO DA TOXICIDADE AGUDA DE PIRACLOSTROBIN, EPOXICONAZOL E SUA MISTURA EM Daphnia similis
}

\author{
ELIZABETH BIAGIONI PRESTES* \\ CLAUDIO MARTÍN JONSSON** \\ VERA LÚCIA S. S. DE CASTRO***
}

\begin{abstract}
No presente trabalho avaliou-se o efeito toxicológico (inibição da mobilidade) de formulações fungicidas à base de piraclostrobin e epoxiconazol, isoladamente e em formulação conjugada sobre Daphnia similis, mediante determinação da Concentração Efetiva Média $\left(\mathrm{CE}_{50}-48 \mathrm{~h}\right)$ de cada uma das formulações. Também foi determinado o possível efeito sinérgico ou antagônico que o uso conjugado dos dois fungicidas poderia exercer em relação à sua toxicidade. Foram encontrados valores de $\mathrm{CE}_{50}-48 \mathrm{~h}$ de 18,36 $\mu \mathrm{g} \cdot \mathrm{L}^{-1}$ para a formulação à base de piraclostrobin, de $89,98 \mu \mathrm{g} \cdot \mathrm{L}^{-1}$ para a formulação à base de epoxiconazol e de 23,50 $\mu \mathrm{g} \cdot \mathrm{L}^{-1}$ para a formulação conjugada. Das três formulações estudadas, o piraclostrobin mostrou-se o mais tóxico para os organismos em estudo. A análise isobolográfica e a determinação do Índice de Aditividade (IA) sugerem que a mistura dos princípios ativos exerce apenas efeito toxicológico aditivo sobre o organismo-alvo.
\end{abstract}

PALAVRAS-CHAVE: PIRACLOSTROBIN; EPOXICONAZOL; TOXICIDADE; Daphnia similis.

* Bióloga, Laboratório de Ecotoxicologia e Biossegurança, Embrapa Meio Ambiente, Jaguariúna, SP, Brasil (e-mail: bbiagioni@gmail.com).

** Farmacêutico, Laboratório de Ecotoxicologia e Biossegurança, Embrapa Meio Ambiente, Jaguariúna, SP, Brasil (e-mail: jonsson@cnpma.embrapa.br).

*** Médica Veterinária, Laboratório de Ecotoxicologia e Biossegurança, Embrapa Meio Ambiente, Jaguariúna, SP, Brasil (e-mail: castro@cnpma.embrapa.br). 


\section{INTRODUÇÃO}

O uso combinado de dois ou mais fungicidas na agricultura vem sendo amplamente realizado devido a uma série de razões: i) aumentar o espectro de ação dos fungicidas para o controle de enfermidades que ocorrem simultaneamente nos cultivos, ii) melhorar a possibilidade de exploração do potencial aditivo e sinérgico dos diferentes princípios ativos, com o aumento geral da eficácia e a redução da concentração dos componentes sem a perda de efetividade; iii) atrasar o desenvolvimento de resistência fúngica a um componente da mistura (GISI, 1996). No mercado brasileiro são encontradas várias formulações combinadas de fungicidas e entre esses os pertencentes às classes dos triazois e estrobilurinas, tais como o epoxiconazol e o piraclostrobin, respectivamente. Esses dois princípios ativos encontram-se amplamente disponíveis em formulações isoladas ou conjugadas entre si.

A ação dos triazois decorre da inibição do esterol 14a-demetilase (codificado pelo gene CYP51) em bolores e leveduras bloqueando a biossíntese do ergosterol, componente essencial das membranas celulares desses organismos (ZARN, BRÜSCHWEILER e SCHLATTER, 2003). Já as estrobilurinas agem inibindo a respiração mitocondrial pela ligação ao citocromo $b$, bloqueando assim a geração de ATP (BARTLETT et al., 2001).

A despeito do seu papel como ferramenta para o aumento da produtividade dos cultivos, os fungicidas apresentam o revés da contaminação de água e solos das áreas de cultivo e adjacências (BATTAGLIN et al., 2011; SILVA et al., 2009) podendo levar à injúria da fauna e flora presentes. Apesar de serem encontrados na literatura trabalhos relativos aos efeitos ecotoxicológicos dos triazois sobre organismos aquáticos, são poucos os estudos sobre as estrobilurinas ou as duas substâncias conjugadas, tampouco são encontrados dados sobre o grau de sinergismo do uso conjugado das duas substâncias sobre tais organismos.

O microcrustáceo planctônico Daphnia similis é amplamente utilizado na avaliação de risco e monitoramento de poluentes em meio aquático, sendo recomendado o seu uso por vários órgãos internacionais (OECD, 2004; EUROPEAN COMMISSION, 2002). Desse modo, no presente trabalho avaliou-se o efeito toxicológico de formulações fungicidas à base de piraclostrobin, epoxiconazol e sua mistura sobre $D$. similis, mediante determinação da Concentração Efetiva $50 \%$ $\left(\mathrm{CE}_{50}-48 \mathrm{~h}\right)$ de cada uma das formulações e a determinação do grau de sinergismo ou antagonismo que a mistura piraclostrobin-epoxiconazol exerce em relação à toxicidade aos organismos em estudo.

\section{MATERIAL E MÉTODOS}

Foram testadas formulações de fungicidas à base de piraclostrobin $(25,0 \% \mathrm{~m} / \mathrm{v})$, epoxiconazol $(12,5 \% \mathrm{~m} / \mathrm{v}$ ) e de sua mistura (piraclostrobin a $13,3 \% \mathrm{~m} / \mathrm{v}$ e epoxiconazol a $5,0 \% \mathrm{~m} / \mathrm{v}$ ), oriundas de apresentações comerciais disponíveis no mercado. Como organismo-teste utilizou-se D. similis com idade inferior a 24 horas. O invertebrado foi previamente cultivado em aquários de $40 \times 25 \times 15 \mathrm{~cm}$, com água declorinizada com as seguintes características físico-químicas: $\mathrm{pH}$ 7,8; dureza total $111,0 \mathrm{mg}$ de $\mathrm{CaCO}_{3} \cdot \mathrm{L}^{-1}$ e condutividade $0,38 \mathrm{mS} . \mathrm{cm}^{-1}$. Os organismos foram mantidos em sala climatizada sob temperatura controlada a $20 \pm 2^{\circ} \mathrm{C}$, luminosidade de 1.000 lux e alimentados com algas das espécies Pseudokirchneriella subcapitata e Chlorella pyrenoidosa.

Os organismos foram expostos por 48 horas a formulações de fungicida à base

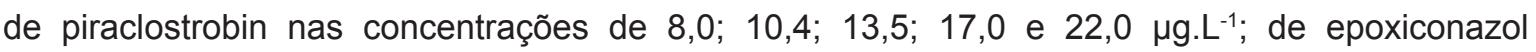
nas concentrações de 20,9;37,0;67,0;120,0 e 220,0 $\mu \mathrm{g} . \mathrm{L}^{-1}$ e da mistura de piraclostrobin e epoxiconazol nas concentrações de 9,$0 ; 17,0 ; 30,8 ; 50,0$ e 100,0 $\mu \mathrm{g} . \mathrm{LL}^{-1}$. Tais soluçõesteste referem-se às concentrações dos ingredientes ativos dissolvidos em água com as características físico-químicas já descritas e oriundas de apresentações comerciais disponíveis no 
mercado.

Os resultados de imobilidade dos organismos face às diferentes concentrações dos agentes químicos foram submetidos à análise de próbitos para a determinação da $\mathrm{CE}_{50}-48 \mathrm{~h}$ e seus respectivos intervalos de confiança a 95\%, utilizando-se o programa Statgraphics Plus (MANUGISTICS, 2001). A análise isobolográfica e o cálculo do Índice de Aditividade, segundo Marking (1977) permitem avaliar a ocorrência de efeito sinérgico, antagônico ou aditivo dos ingredientes na mistura sobre o organismo-teste. Determinou-se o grau de magnificação desse efeito conforme procedimento descrito por Marking (1985).

\section{RESULTADOS E DISCUSSÃO}

No presente trabalho, o piraclostrobin apresentou a maior toxicidade à $D$. similis entre os fungicidas testados, seguido pela mistura (piraclostrobin + epoxiconazol) e pelo epoxiconazol, sendo os três valores de $\mathrm{CE}_{50}-48 \mathrm{~h}$ estatisticamente diferentes entre si (Tabela 1).

Segundo a classificação da Agência de Proteção Ambiental dos Estados Unidos (U.S. EPA, 1985), compostos com valores de $\mathrm{CE}_{50}-48 \mathrm{~h}$ inferiores a $100 \mu \mathrm{g} \cdot \mathrm{L}^{-1}$ são considerados de "extrema toxicidade". Os dados obtidos neste trabalho enquadram os fungicidas estudados, assim como sua mistura, nessa categoria.

\section{TABELA 1 - PARÂMETROS DE TOXICIDADE AGUDA PARA D. similis EXPOSTAS A DIFERENTES CONCENTRAÇÕES DE PIRACLOSTROBIN, EPOXICONAZOL E SUA MISTURA POR 48 HORAS}

\begin{tabular}{|c|c|c|c|c|}
\hline Fungicida & $\begin{array}{c}C E_{50}-48 h \\
\left(\mu g \cdot L^{-1}\right)\end{array}$ & $\begin{array}{c}\text { Intervalo de } \\
\text { confiança }\left(\mu \mathrm{g} \cdot \mathrm{L}^{-1}\right)\end{array}$ & $\begin{array}{l}\text { Modelo de regressão } \\
\qquad y=\text { normal (eta) }\end{array}$ & $\mathbf{R}^{2}(\%)$ \\
\hline Piraclostrobin & 18,36 & $16,78-20,58$ & eta $=-3,6981+201,384 x$ & 75,26 \\
\hline Epoxiconazol & 89,98 & $76,13-109,30$ & eta $=-2,20004+24,4495 x$ & 84,10 \\
\hline $\begin{array}{c}\text { Formulação } \\
\text { (Piraclostrobin + } \\
\text { Epoxiconazol) }\end{array}$ & 23,50 & $20,44-27,04$ & eta $=-3,51811+149,701 x$ & 91,43 \\
\hline
\end{tabular}

Após 48 horas de exposição ao piraclostrobin, o grupo tratado com $22 \mu \mathrm{g} \cdot \mathrm{L}^{-1}$ do agente químico apresentou taxa de imobilidade dos organismos testados de $75 \%$, valor significativamente maior que os demais tratamentos, excetuando-se o grupo tratado com $17 \mu \mathrm{g} \cdot \mathrm{L}^{-1}$, conforme observado na Figura 1.

Face aos resultados obtidos, a $\mathrm{CE}_{50}-48 \mathrm{~h}$ do piraclostrobin em relação à $D$. similis foi de 18,36 $\mu \mathrm{g} \cdot \mathrm{L}^{-1}$ (Tabela 1).

Em trabalho com D. magna, Ochoa-Acuña et al. (2009) encontraram para o piraclostrobin $\mathrm{CE}_{50}-48 \mathrm{~h}$ de $68 \mu \mathrm{g} \cdot \mathrm{L}^{-1}$ e para $96 \mathrm{~h}$ de $14 \mu \mathrm{g} \cdot \mathrm{L}^{-1}$. Um relatório do governo australiano, avaliando o piraclostrobin em apresentação comercial informa $\mathrm{CE}_{50}-48 \mathrm{~h}$ de $15,7 \mu \mathrm{g} \cdot \mathrm{L}^{-1}$, valor bastante próximo ao encontrado no presente trabalho (AUSTRALIAN PESTICIDES AND VETERINARY MEDICINES AUTHORITY, 2003). 


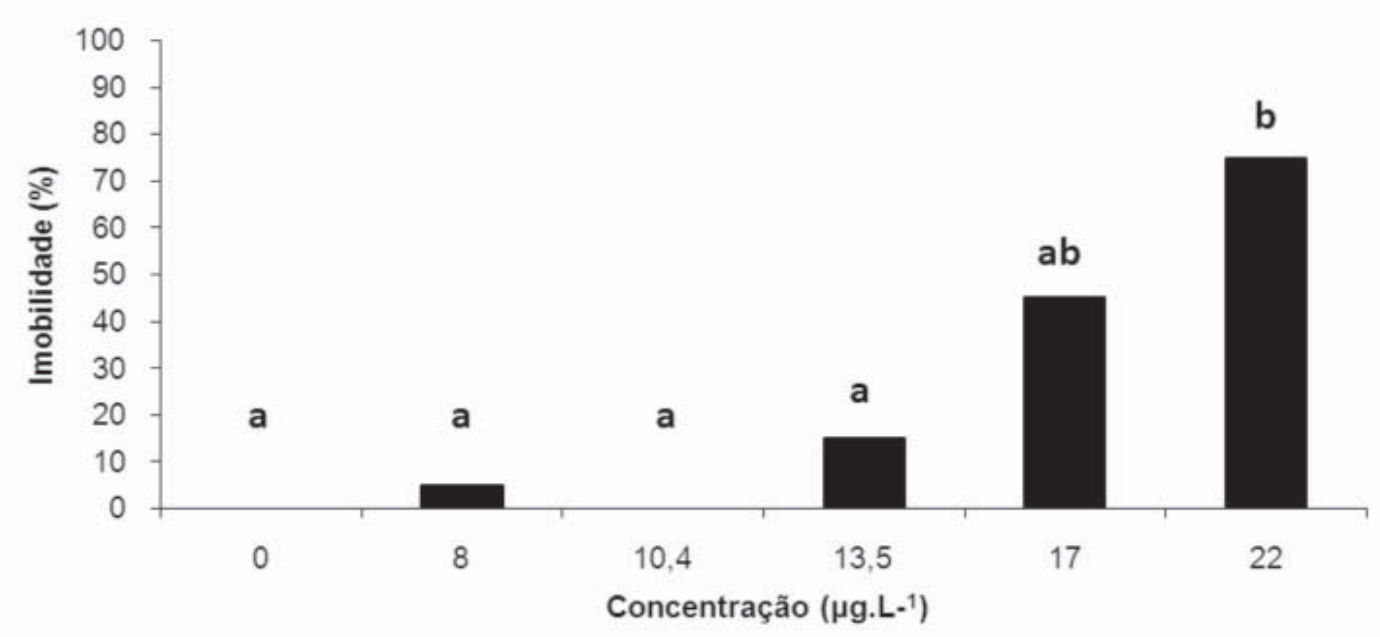

\section{FIGURA 1 - PORCENTAGEM DE IMOBILIDADE DE D. similis SUBMETIDAS A DIFERENTES CONCENTRAÇÕES DE PIRACLOSTROBIN POR 48 HORAS}

Letras diferentes indicam diferença significativa $(p<0,05)$.

Godwin et al. (2000), em trabalho sobre a toxicidade de outra estrobilurina (picoxistrobin) sobre D. magna obtiveram $\mathrm{CE}_{50}-48 \mathrm{~h}$ de $18 \mu \mathrm{g} \cdot \mathrm{L}^{-1}$, semelhante ao valor encontrado neste trabalho.

Após 48h de exposição ao epoxiconazol, o grupo tratado com $220 \mu \mathrm{g} \cdot \mathrm{L}^{-1}$ do agente químico apresentou taxa de imobilidade dos organismos testados de $100 \%$, seguido pelo grupo tratado com $120 \mu \mathrm{g} . \mathrm{L}^{-1}$ que revelou $80 \%$ de imobilidade. Esses dois grupos não apresentaram diferenças significativas entre si, entretanto foram significativamente diferentes de todos os demais grupos, os quais não apresentaram taxa de imobilidade maior que 20\% (Figura 2).

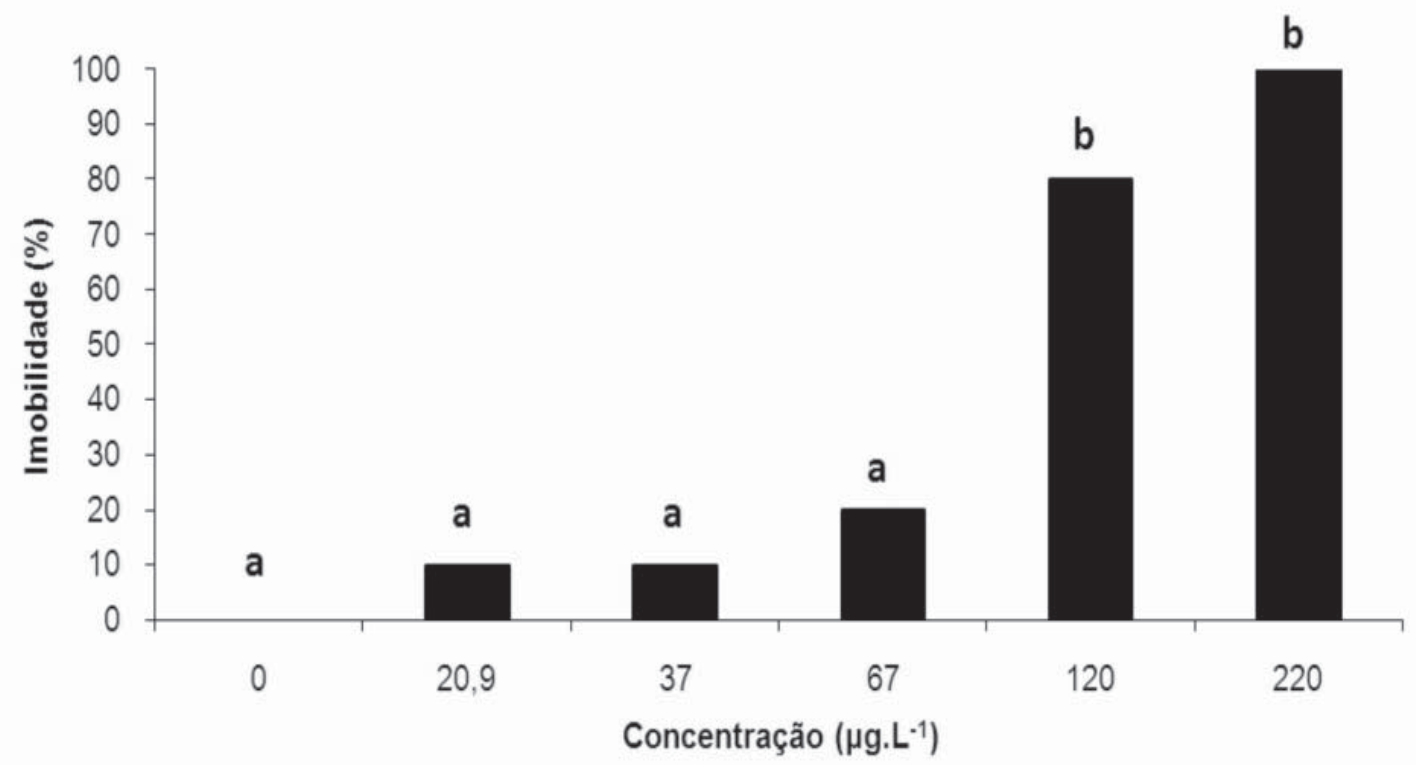

FIGURA 2 - PORCENTAGEM DE IMOBILIDADE DE D. similis SUBMETIDAS A DIFERENTES CONCENTRAÇÕES DE EPOXICONAZOL POR 48 HORAS

Letras diferentes indicam diferença significativa $(p<0,05)$. 
No presente trabalho, a $\mathrm{CE}_{50}-48 \mathrm{~h}$ do fungicida epoxiconazol em relação a $D$. similis foi de $89,98 \mu \mathrm{g} . \mathrm{L}^{-1}$ (Tabela 1). Valores diferentes, $\mathrm{CE}_{50}-48 \mathrm{~h}$ de $8.700 \mu \mathrm{g} \cdot \mathrm{L}^{-1}$, foram encontrados por Probst et al. (2005) para epoxiconazol em D. magna. É sabido que diferentes espécies do gênero Daphnia podem apresentar sensibilidade distinta frente aos agentes estressores (BAIRD et al., 1989).

Após 48h de exposição à mistura de piraclostrobin e epoxiconazol, os grupos tratados com 50 e $100 \mu \mathrm{g} \cdot \mathrm{L}^{-1}$ do agente químico apresentaram taxa de imobilidade dos organismos testados de $100 \%$, seguidos pelo grupo tratado com $30,8 \mu \mathrm{g} . \mathrm{L}^{-1}$ que revelou $85 \%$ de imobilidade. Esses três grupos não apresentaram diferenças significativas entre si, entretanto foram significativamente diferentes dos demais grupos que não evidenciaram taxa de imobilidade maior que $20 \%$ (Figura 3).

A $\mathrm{CE}_{50}-48 \mathrm{~h}$ encontrada para a mistura piraclostrobin e epoxiconazol foi de $23,50 \mu \mathrm{g} . \mathrm{L}^{-1}$ (Tabela 1). Entretanto, a escassez de trabalhos disponíveis na literatura com a utilização da mistura dificulta a comparação dos resultados. O fabricante de uma das apresentações comerciais, cuja formulação constitui-se de piraclostrobin $(12,5 \% \mathrm{~m} / \mathrm{m})+$ epoxiconazol $(4,7 \% \mathrm{~m} / \mathrm{m})$, divulga $\mathrm{CE}_{50}-48 \mathrm{~h}$ de $100 \mu \mathrm{g} . \mathrm{L}^{-1}$, não citando a espécie de Daphnia utilizada (BASF, 2009). Apresentação comercial do mesmo fabricante com a associação de epoxiconazol $(11,5 \% \mathrm{~m} / \mathrm{m})$ e a estrobilurina cresoximmetílico $(11,5 \% \mathrm{~m} / \mathrm{m})$ informa $\mathrm{CE}_{50}-48 \mathrm{~h}$ de $730 \mu \mathrm{g} \cdot \mathrm{L}^{-1} \mathrm{em}$ estudo com Daphnia magna (BASF, 2003). A mesma associação em diferentes porcentagens $(12,5 \% \mathrm{~m} / \mathrm{v}$ de epoxiconazol e $12,5 \% \mathrm{~m} / \mathrm{v}$ de cresoxim-metílico), oriunda de outro fabricante, tem $\mathrm{CE}_{50}-72 \mathrm{~h}$ divulgada de $1.380 \mu \mathrm{g} . \mathrm{L}^{-1}$ para a mesma espécie (MILENIA AGROCIÊNCIAS, 2007).

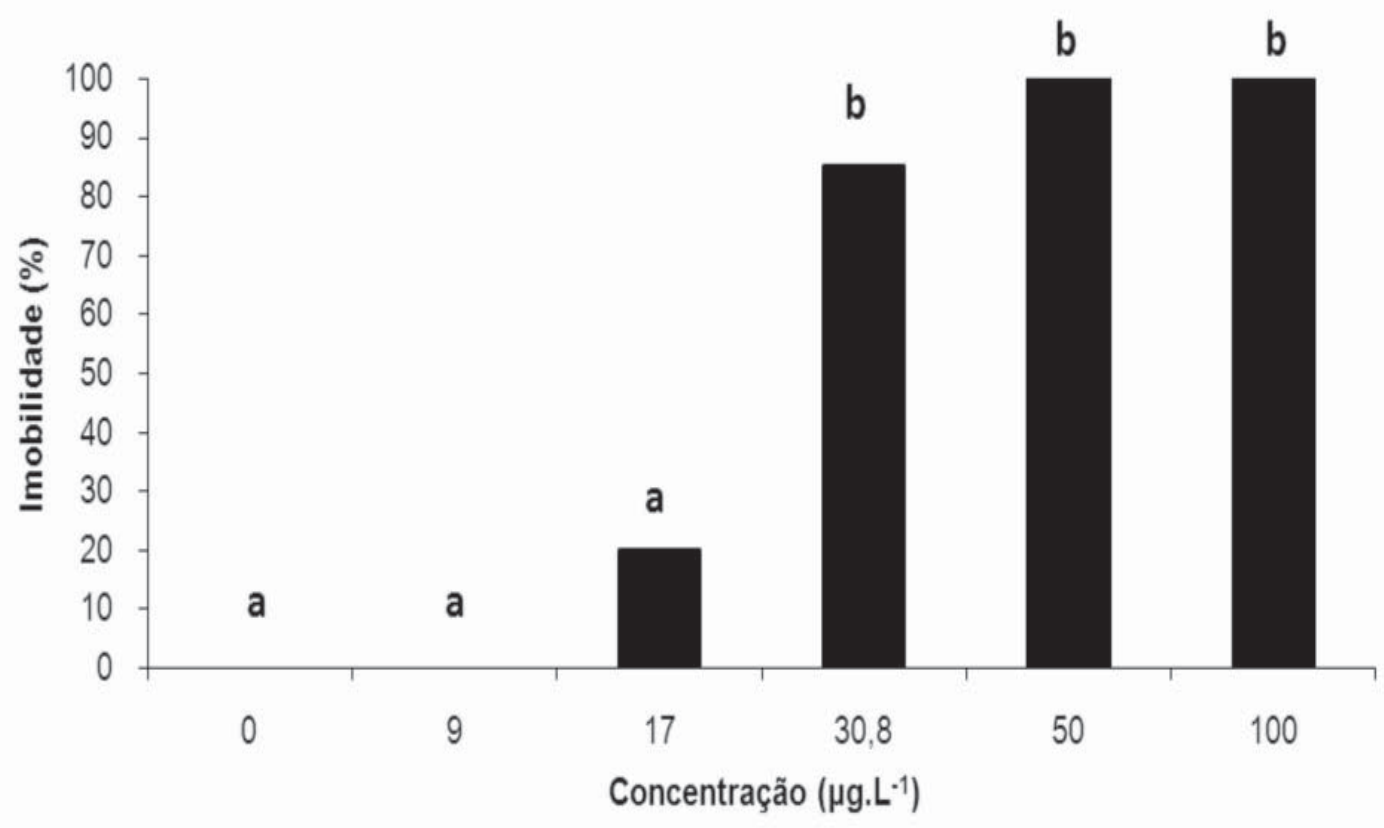

\section{FIGURA 3 - PORCENTAGEM DE IMOBILIDADE DE D. similis SUBMETIDAS A DIFERENTES CONCENTRAÇÕES DA MISTURA (PIRACLOSTROBIN + EPOXICONAZOL) POR 48 HORAS}

Letras diferentes indicam diferença significativa $(p<0,05)$.

Os resultados encontrados no presente trabalho indicam maior toxicidade da formulação conjugada (piraclostrobin + epoxiconazol) à Daphnia que as referenciadas na literatura. Fatores como alimentação, espécies utilizadas, variações entre os diferentes clones e características físicoquímicas da água, entre outros, já foram relatados como potenciais causadores de variação na sensibilidade desses organismos frente a agentes estressores (PAULAUSKIS \& WINNER, 1988; BAIRD et al., 1989; PEREIRA \& GONÇALVES, 2007). 
O cálculo do Índice de Aditividade (IA) resultou em valor igual a 0, estimando-se, que não há acréscimo ou diminuição relevante da toxicidade da mistura em relação à de cada composto individualmente. O isobolograma (Figura 4) também sugere que o uso da formulação conjugada entre piraclostrobin e epoxiconazol não traz efeito sinérgico ou antagônico relevante em relação à sua ação tóxica sobre o organismo-teste, quando comparado às formulações isoladas. Tal resultado indica apenas efeito aditivo entre os fungicidas.

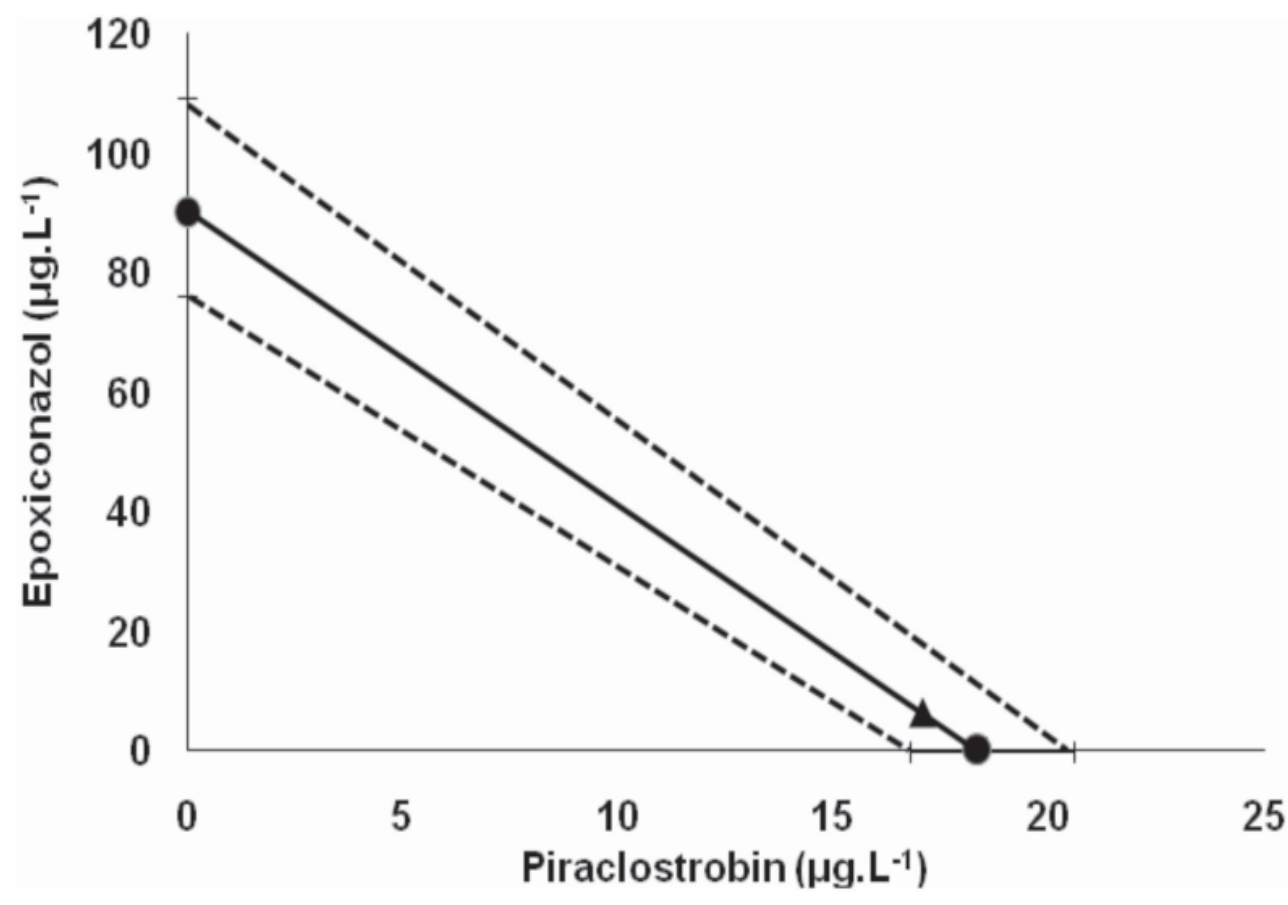

\section{FIGURA 4 - ANÁLISE ISOBOLOGRÁFICA PARA PIRACLOSTROBIN E EPOXICONAZOL}

A figura triangular indica a concentração da combinação piraclostrobin + epoxiconazol que causa $50 \%$ de inibição da mobilidade. O Índice de Aditividade (IA) calculado para esse ponto equivale a 0.

\section{CONCLUSÃO}

O fungicida piraclostrobin apresentou maior efeito tóxico à $D$. similis em relação ao epoxiconazol ou à mistura de ambos.

A variabilidade entre alguns resultados de $\mathrm{CE}_{50}-48 \mathrm{~h}$ presentes na literatura e os encontrados neste trabalho pode estar associada a variações nas espécies utilizadas, origem dos clones, regime de alimentação e água, entre outros fatores, que podem influenciar a sensibilidade de Daphnia frente a agentes estressores.

O uso conjugado das duas substâncias não resultou em efeito sinérgico no que tange à toxicidade a $D$. similis. $O$ Índice de Aditividade sugere apenas efeito aditivo entre os dois compostos.

Os resultados do presente estudo são úteis para expandir a base de dados referente a efeitos de fungicidas e suas misturas sobre organismos não-alvo. 


\section{ABSTRACT \\ TOXICITY OF FORMULATIONS BASED ON PIRACLOSTROBIN, EPOXICONAZOLE AND ITS COMBINATION TO Daphnia similis}

The current paper has investigated toxicological effects of fungicidal formulations based on piraclostrobin, epoxiconazole and its combined formulations to Daphnia similis through determination of Mean Effective Concentration (EC50-48h) from each formulation. It was also determined the synergistic/antagonic effect that the combined use of two fungicides could have over its toxicity. The EC50-48h values observed were 18.36 $\mu$ g. $\mathrm{L}^{-1}$ for piraclostrobin; $89.98 \mu \mathrm{g} . \mathrm{L}^{-1}$ for epoxiconazole and $23.50 \mu \mathrm{g} . \mathrm{L}^{-1}$ for combined fungicides formulation. Among the three studied formulas, piraclostrobin was the most toxic fungicide for the studied organisms. Isobolographic analysis and Additivity Index suggest that the combined use of two fungicides in a single formulation only exerts additive toxicological effect on target organism

KEY-WORDS: PIRACLOSTROBIN; EPOXICONAZOLE; TOXICITY; Daphnia similis.

\section{REFERÊNCIAS}

1 AUSTRALIAN PESTICIDES AND VETERINARY MEDICINES AUTHORITY (APVMA). Evaluation of the new active pyraclostrobin in the product Cabrio fungicide. Canberra, 2003.

2 BAIRD, D.J.; BARBER, I.; BRADLEY, M.; CALOW, P.; SOARES, A.M.V. M. The Daphnia bioassay: a critique. Hydrobiologia, Dordrecht, v. 188-189, n. 1, p. 403-406, 1989.

3 BARTLETT, D.W.; CLOUGH, J.M.; GODFREY, C.R.A.; GODWIN, J.R.; HALL, A.A.; HEANEY, S.P.; MAUND, S.J. Understanding the strobilurin fungicides. Pesticide Outlook, London, v. 4, n. 12, p. 143-148, 2001.

4 BASF NEW ZEALAND LIMITED. Allegro ${ }^{\circledR}$. Safety Data Sheet. Auckland, 2003. (Código do documento, NZ 6262).

5 BASF CROP PROTECTION. Opera ${ }^{\circledR}$. Prospecto de produto. Cheadle, Cheshire, 2009. (Código de documento: $\sim$ Xmam1249027715.indd).

6 BATTAGLIN, A.W.; SANDSTROM, M.W.; KUIVILA, K.M.; KOLPIN, D.N.; MEYER, M.T. Occurrence of azoxystrobin, propiconazole and selected other fungicides in US streams, 2005-2006. Water, Air, \& Soil Pollution, Dordrecht, v. 218, n. 1-4, p. 307-322, 2011

7 EUROPEAN COMMISSION. Guidance document on aquatic ecotoxicology. rev. $4^{\text {th }}$ Brussels, 2002.

8 GISI, U. Synergistic interaction of fungicides in mixtures. Phytopathology, Ithaca, v. 86, n. 11, p. 1273-1279, 1996.

9 GODWIN, J.R.; BARTLETT, D.W.; CLOUGH, J.M.; GODFREY, C.R.A.; HARRISON, E.G.; MAUND, S.J. Picoxystrobin: a new strobilurin fungicide for use on cereals. In: BCPC CONFERENCE - PESTS AND DISEASES, $2^{\text {th }}, 2000$. Proceedings... Brighton: BCPC, 2000. p. $533-540$.

10 MANUGISTICS Statgraphics Plus. Version 5.1 for Windows. Rockville: Manugistics Group, 2001.

11 MARKING, L.L. Method for assessing additive toxicity of chemical mixtures. In: MAYER, F.L.; HAMELINK, J.L. (Org.). Aquatic toxicology and hazard evaluation. Philadelphia: American Society for Testing and Materials, 1977, p. $99-18$.

12 MARKING, L. L. Toxicity of chemical mixtures. In: RAND, G.M.; PETROCELLI, S.R. (Org.). Fundamentals of aquatic toxicology: methods and applications. Washington: Hemisphere, 1985. p.164-176.

13 MILENIAAGROCIÊNCIAS. Guapo ${ }^{\circledR}$. Ficha de informações e segurança de produtos químicos. Londrina, 2007. (Revisão de documento, 1).

14 OCHOA-ACUÑA, H.G.; BIALKOWSKI, W.; YALE, G.; HAHN, L. Toxicity of soybean rust fungicides to freshwater algae and Daphnia magna. Ecotoxicology, London, v. 18, p. 440-446, 2009.

15 OECD. Organization for Economic Co-operation and Development. Guideline for testing on chemicals: Daphnia sp. acute immobilization test. Paris, 2004.

16 PAULAUSKIS, J.D.; WINNER, R.W. Effects of water hardness and humic acid on zinc toxicity to Daphnia magna Straus. Aquatic Toxicology, Amsterdam, v. 12, n. 3, p. 273-290, 1988.

17 PEREIRA, J.L.; GONÇALVES, F. Effects of food availability on the acute and chronic toxicity of the insecticide methomyl 
to Daphnia spp. Science of the Total Environment, Amsterdam, v. 386, n. 1-3, p. 9-20, 2007.

18 PROBST, M.; BERENZEN, N.; LENTZEN-GODDING, A.; SCHULZ, R. Scenario-based simulation of runoff-related pesticide entries into small streams on a landscape level. Ecotoxicology and Environmental Safety, New York, v. 62, p. 145-159, 2005.

19 SILVA, D.R.O.; AVILA, L.A.; AGOSTINETTO, D.; DAL MAGRO, T.; OLIVEIRA, E.; ZANELLA, R.; NOLDIN J. A. Monitoramento de agrotóxicos em águas superficiais de regiões orizícolas no sul do Brasil. Ciência Rural, Santa Maria, v.39, n. 9, p. 2383-2389, 2009.

20 U.S. ENVIRONMENTAL PROTECTION AGENCY (U.S.EPA). Hazard Evaluation Division. Standard evaluation procedure: acute toxicity test for freshwater invertebrates. Washington, D.C., 1985.

21 ZARN, A.J.; BRÜSCHWEILER, B.J.; SCHLATTER, J.R. Azole fungicides affect mammalian steroidogenesis by inhibiting sterol 14 alpha-demethylase and aromatase. Environmental Health Perspectives, Durham, v.111, n.3, p.255-261, 2003.

\section{AGRADECIMENTOS}

Os autores agradecem ao Conselho Nacional de Desenvolvimento Científico e Tecnológico (CNPq) e à Fundação de Amparo à Pesquisa do Estado de São Paulo (FAPESP) pelo suporte financeiro ao estudo. 